\title{
STRATEGIC PLAN FOR SCIENTIFIC COMPUTING (U)
}

by J.P. Church

Westinghouse Savannah River Company

Savannah River Site

Aiken, South Carolina 29808

Other Authors:

\section{RECEIVED \\ FEB - 51993 \\ OSTI}

This paper was prepared in connection with work done under Contract No. DE-AC09-89SR18035 with the U. S. Department of Energy. By acceptance of this paper, the publisher and/or recipient acknowledges the U.S. Government's right to retain a nonexclusive, royalty-free license in and to any copyright covering this paper, along with the right to reproduce and to authorize others to reproduce all or part of the copyrighted paper.

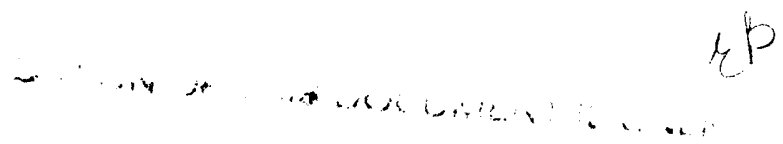




\section{DISCLAIMER}

This report was prepared as an account of work sponsored by an agency of the United States Government. Neither the United States Government nor any agency thereof, nor any of their employees, makes any warranty, express or implied, or assumes any legal liability or responsibility for the accuracy, completeness, or usefulness of any information, apparatus, product, or process disclosed, or represents that its use would not infringe privately owned rights. Reference herein to any specific commercial product, process, or service by trade name, trademark, manufacturer, or otherwise does not necessarily constitute or imply its endorsement, recommendation, or favoring by the United States Government or any agency thereof. The views and opinions of authors expressed herein do not necessarily state or reflect those of the United States Government or any agency thereof.

This report has been reproduced directly from the best available copy.

Available to DOE and DOE contractors from the Office of Scientific and Technical Information, P. O. Box 62, Oak Ridge, TN 37831; prices available from (615) $576-8401$.

Available to the public from the National Technical Information Service, U. S. Department of Commerce, 5285 Port Royal Rd., Springfield, VA 22161. 
DOCUMENT: WSRC-TR-92-235

TITLE: STRATEGIC PLAN FOR SCIENTIFIC COMPUTING (U)

AUTHOR: J. P. CHURCH

\section{APPROVALS}

M. R. BUCKNER, MANAGER

SCIENTIFIC COMPUTATIONS SECTION

DATE:

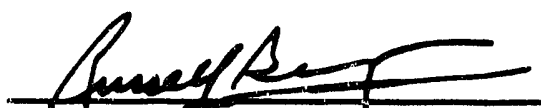

R. R. BECKMEYER, MANAGER

$05 / 19 / 92$ COMPUTING TECHNOLOGY GROUP 
IABLE OF CONTENTS

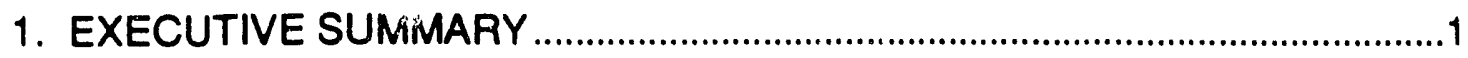

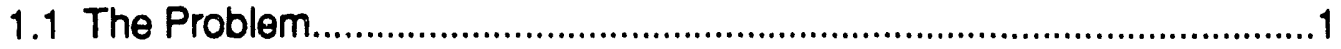

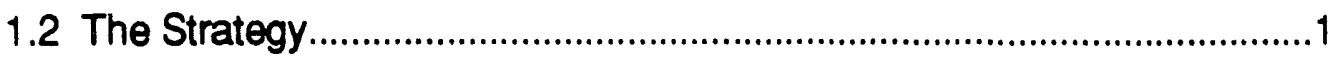

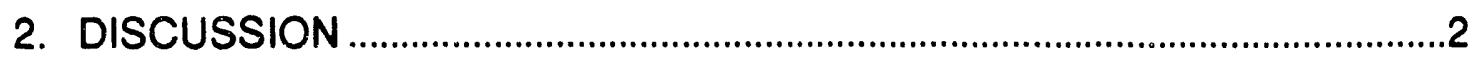

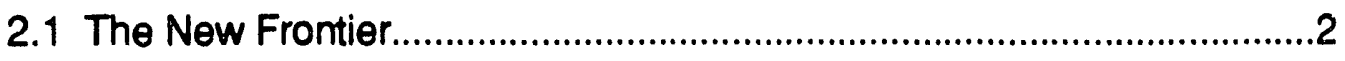

2.2 The Computing Environment Problem...................................................

2.3 The Problem of Rapidly Changing Technology .................................

2.4 The Solution ('The SCEED that grows.') ..............................................

2.4.1 Description ..................................................................................

2.4.2 High Speed Backplane ...........................................................

2.4.3 Compute Servers.........................................................................

2.4.4 File/Print/Security/Administrative Servers.............................

2.4.5 Archive/Backup Server .........................................................6

2.4.6 Visualization Servers............................................................

2.4.7 User Interface .............................................................................

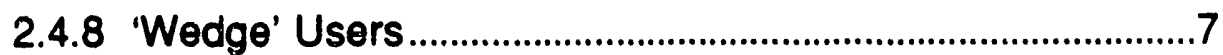

2.4.9 Status .....................................................................................

2.5 The Other Parts of the Strategy ..........................................................

2.5.1 Alliances with DOE and NSF Supercomputer Centers......8

2.5.1.1 NSF Supercomputing Centers .................................

2.5.1.2 DOE Supercomputing Centers .................................

2.5.2 Site Connection to the Internet.................................................10

2.5.3 Massively Parallel Architecture Computer .............................11

2.5.3.1 Programmatic Need .................................................12

2.5.3.1.1 Physics Calculations ..................................13

2.5.3.1.2 Environmental Program Calculations .....13

2.5.3.2 Supercomputer Upgrade.........................................14 


\section{STRATEGIC PLAN FOR SCIENTIFIC COMPUTING}

1.

EXECUTIVE SUMMARY

1.1

The Problem

Computing technology continues to undergo rapid and dramatic changes. Technological improvements in both hardware and software continue to permit analysts to model problems much more realistically than heretofore practicable. New visualization technologies vastly increase our ability to understand the results of those complex models.

The mission of SRS is also undergoing very rapid change as a result of international events. While the typical demands of reactor oriented calculations may decline, environmental regulations require us to study new classes of problems in ever increasing detail. Hence, the computational workload is actually increasing rapidly. At the same time, the budget constraints demand a continued increase in cost effectiveness of scientific computing.

A comprehensive strategy for scientific computing is required to adapt to these changes and still produce timely solutions to ensure continued safe operation of SRS facilities. An important goal of this strategy is to ensure that productivity gains available with new systems and technologies are truly achieved.

\subsection{The Strategy}

A strategy for scientific computing is defined comprising four interrelated components.

(1) Build a small modern integrated system, initially based on a high-speed network of high-performance workstations, as a fully implemented production environment demonstration with easy access from the user's desktop via local and wide area networks. Integrate the existing computational infrastructure with the new system as the existing components acquire the required technologies. Distributed computing and coarse grained parallelism will be probed with this small integrated system.

(2) Collaborate with DOE and NSF Supercomputer Centers. These sites are involved in advanced supercomputing research and development, and each has an emphasis in a particular phase of high performance computing, communication, and visualization. Guidance from, and cooperation with, these sites will minimize the task of implementing the desired computational capabilities at SRS.

(3) Connect to Internet, the national high-speed network linking major computing facilities in the country. This connection helps SRS interact with the offsite technical community and enhances opportunities for collaborative R\&D. Access to supercomputing cycles is also available through this connection.

(4) Make available, first through the Internet and then by acquiring onsite as needed, a massively parallel architecture computer. This technology will allow increasingly 
sophisticated analyses to be completed fast and cheaply enough to consider icr a greater proportion of the SRS studies.

\section{DISCUSSION}

\section{$2.1 \quad$ The New Frontier}

Computational scientists are at the frontier of a new era in computing. The rapidly developing computing technology, both in hardware and software, promises to change the way we think about, let alone solve, problems. The major strides made in $3 \mathrm{D}$ visualization and in Virtual Reality (a technique for putting the scientist virtually 'inside' the problem), allow the scientist to routinely view the problem domain in its full dimensionality. The impact of this will be enormous. Every problem in the real world is at least four-dimensional (three spatial dimensions plus time dependence). But until relatively recently (last 10 years), a full 3D time-dependent solution (i.e., 4D) was prohibitively expensive and, thus, not attempted for any but the most critical problems.

Instead, scientists have historically devised and solved one- and two-dimensional analogs to the real 3D problem. And only a few (usually two: beginning-, and end-, ofstate) static cases were run to indicate the range of results to be expected. At best, the analogs were an excellent approximation to the real problem, and corrections could be made to account for the remaining 3D effects. At worst, the solutions gave meager guidance about how to apply the results to the real problem. Frequently, the beginning-, and end-, of-state results gave no indication of intermediate cases having results that exceeded the end points by a substantial amount.

But as computational power has increased, full 3D solutions can be afforded and obtained for more and more applications preblems. The resulting insight into the real problems and their solutions promises a revolution in understanding of the world. In fact, this computer simulation of reality is being called "the third branch of science".1 The visualization of multidimensional ( $n D, n \geq 3$ ), perhaps more easily thought of as multiparametric, results was until fairly recently only achievable in supercomputer centers specializing in computer graphics. However, the rapid reduction in cost of computer hardware has broadened the availability of the graphics technology. We are virtually at the threshold of routinely using computer graphics to display and facilitate understanding the results of 4D calculations of radionuclide contaminant plumes in groundwater flow models or atmospheric releases, onset of boiling inside multitube reactor assemblies, fission power densities in heterogeneous lattices made up of several different types of assemblies, or the migration of hydrogen through a metal. Visualizing such information, which may be facilitated by animation and sonification, is crucial to understanding and refining the overall fidelity of the computational model.

These important techniques require very high rates of transfer of very large amounts of data and demands improved computing facilities. The concomitant improvements in computing facilities necessary for SRS to be able to perform such modeling complexities are discussed in the strategic plan presented here. All pieces of the plan

1 "Computing in Science". Science, pp. 44-64, Vol.256, Number 5053, April 3, 1992. 
are necessary to support site studies and plans to achieve success in, for example, compliance with environmental regulations. The discussion below further explains the problems of working within the present environment and outlines the advantages of moving to the proposed environment.

\subsection{The Comouting Environment Problem}

An SRS scientist or engineer who tries to optimally use existing computing facilities is severely hampered by barriers that prevent easy use of the overall system. These barriers are not unique to SRS, and they occur because each system (IBM mainframe. Cray XMP-EA, VAX cluster, or desktop workstation) uses a separate operating system. Although each piece of the system provides excellent capabilities within its respective original design goals, the pieces are not fully integrated into a seamless transparent system. Separate rules exist for using each system. Moving data between systems requires awkward, non-intuitive steps and recipes.

The overall effect is to make the scientist/engineer user extremely reluctant to use new systems, methods, architectures, and technologies. Productivity gains available with new technology are slowly, if ever, achieved because insufficient resources are committed to administer the resulting systems in a cohesively integrated way. Such a commitment would, until relatively recent advances in commercial software became available, have required major staff augmentation to write custom software. It would also have required significant rewrites of that software whenever new versions of the various operating systems were released.

On the hardware side, the traditional way to upgrade the existing computational system requires major capital expenditures to acquire a significant increase in capacity or compute-cycles. There is no way to continuously upgrade the existing site computing facilities with incremental increases in capacity. By the very nature of the existing system architecture, significant improvements in capacity have required large mainframe replacements or additions.

What is needed is a system, and a plan for implementing that system, that provides flexibility to respond to rapidly changing technology, while meeting the needs for continual growth in both productivity (do more with the horsepower we have) and computing cycles (get more horsepower). These needs are driven, in turn, by the ever increasing needs for more sophisticated modeling and data analysis for all aspects of SRS operation, from physics applications to waste disposal, from emergency response to environmental restoration. In addition, the system and plan must accommodate budget constraints that can be swiftly changed in response to rapidly changing international conditions.

\subsection{The Problem of Rapidly Changing Technology}

The rate of change in the computer technology field is incredibly fast. Even more significant is the fact that the rate of change is itself increasing. The U.S. Office of Science and Technology forecasts, and a goal of the Federal High Performance 
Computing and Communication (HPCC) Program is to achieve. a thousand-fold increase in computing capability by 1996.2 Such rapid rates of change in capability challenge the entire method of approach to solving computational problems.

A strategy for scientific computing is needed to facilitate the scientific user's coping with, and adapting to, that rapi. I rate of change. In developing this strategy, we recognize that the key to the ability to respond to change is a core set of principles that remains constant. In this case, the scientific user needs a consistent universe, or user interface, to interact with the various computer platforms. The implementation of that interface is discussed in the following section.

The Computer Architecture and Standards Team has previously published recommendations for the SRS computing architecture ${ }^{3}$ and a guide for migrating to that architecture ${ }^{4}$. These documents summarized the trends in both the needs of the site (increasing demands for sharing of resources, including hardware, software and data) and the technology for computing (increasing use of high performance workstations, distributed computing over high speed networks, and increasing use of UNIX operating systems). The recommendations, which have been approved as site goals, include the following:

1. Pilot projects be done using Distributed Computing Environment.

2. Systems and workstations be closely integrated into the slie computer network with supporting services.

3. Maintain robust mission-essential computing facilities.

These recommendations address the concern of increased productivity. The strategy for scientific computing implements these specific recommendations, and, in addition, provides a means of increasing the actual computing cycles (more, or better, hardware). The components of the first part of the strategy are encompassed in the discussion below of the SCEED system. The components of the second part of the strategy are then discussed. These latter include: (1) establishing alliances with DOE and NSF Supercomputer Centers; (2) establishing site connection to the Internet, the national high-speed network interconnecting major computing facilities in the country; (3) making available, first through the Internet and then by acquiring onsite as needed, a massively parallel architecture computer, most probably of scalable design in keeping with the scalability of the SCEED system discussed next.

\subsection{Ihe Solution ('The SCEED that arows.')}

The first part of the strategy for scientific computing is to build a small modern integrated system as a fully implemented production environment demonstration (a seed that receives all the nutrients necessary to be a healthy, vigorous system), and

2 Grand Challenges: Hiah Performance Computing and Communications, A Report by the Committee on Physical, Mathematical, and Engineering Sciences, Office of Science and Technology Policy, 1992.

3 Savannah River Site Computing Architecture (U), WSr,U-IM-91-18-1 (April 15, 1991).

4 Savannah River Site Computing Architecture Migration Guide (U), WSRC-IM-91-18-2 (July, 30, 1991). 
then integrate the existing system with the new system (i.e., 'grow' the seed) as the existing components acquire the required technologies.

This task, called the Scientific Computing Environment Evaluation Demonstration (SCEED) system, will provide a new production environment using the latest technology for distributed computing. 5 it is a necessary part of upgrading the site's Class VI/Category R computing capability. It is not, however, a replacement for a supercomputar capability. Rather, it is a necessary first step to developing for SRS a meta-computer6 that: (1) makes the residual capacity of participating workstations available as a site resource for predominantly scalar applications, and (2) makes the site mainframe vector and multiprocessor capabilities, as well as the site file storage and backup facilities, available via uniform interfaces. When SCEED is completed, it will be integrated with the existing scientific computing infrastructure as those components acquire the appropriate technology.

\subsubsection{Description}

The initial configuration of the proposed SCEED system is diagrammed in Figure 1. Other configurations can be designed to take advantage of entianced network capabilities and designs as they become available.

This initial configuration comprises a very high speed backplane to which low-end and high-end compute servers (CS), file servers (FS), archive and backup servers, and visualization servers (VS) are connected. Users login to the system via the Local Area Network (LAN) connected to the low-end compute servers. The user's interface is any platform running an $X$ Window System Server.

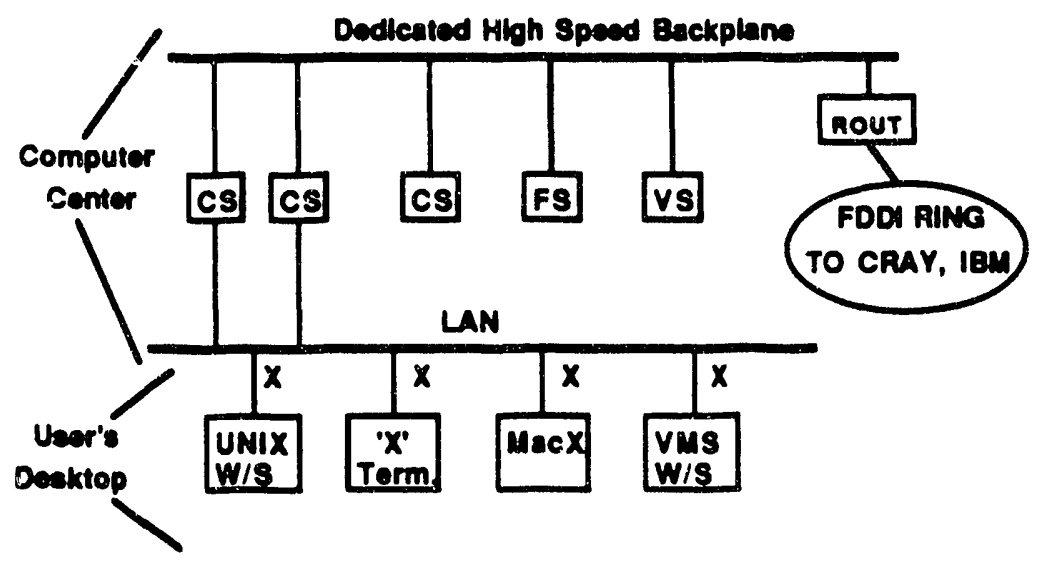

Figure 1. Example Configuration of Scientific Computing Environment Evaluation and Development (SCEED) System

5 J. P. Church. Description of Proposed Scientific Computing Environment Evaluation and Development Project (SCEED). SCS-PMG-920021 (March 18, 1992).

6 'Meta-computer' is a concept of a timesharing network of supercomputers and high-speed storage devices to which a user submits a task. The component of the metacomputer that is used for a specific task can either be specified by the user, or he may instead specify requirements for memory, speed, etc. and the system suppolies a componem that meets those needs.. 
The proposed system is sized to accommodate a limited number of concurrent users and developers. The components of the proposed system and their respective requirements are listed below. A key specification of these components is that they all must use the same version of the same operating system. All compute, file, and archive servers must initially be the same architecture and from the same vendor. Questions of heterogeneity and scalability will be addressed in later phases of the project as SCEED is integrated with the existing computing and networking facilities. A key part of this plan is to adopt open systems and standards. This will enable SRS computational scientists to take advantage of the latest software and hardware developments and make it easy for users to implement them.

\subsubsection{High Speөd Backplane}

A dedicated high-speed backplane, linked to existing SRTC/CCF high speed network, will be provided for maximum data transfer rates. Initially, Ethernet will be used to quickly get the system up and running. However, an upgrade to Fiber Distributed Data Interiace (FDDI) is already funded for the SRTC computer center and will provide greatly enhanced data transfer rates as soon as installed in SCEED. Subsequent upgrades to High-Performance Parallel Interface (HIPPI) or Fiber Channel Standard are planned.

\subsubsection{Compute Servers}

Both lowy-end and high-end compute servers will be provided. The initial configuration uses the low-end servers for usei interfares and for those services that are not so compre intensive. The high-end connute servers will provide the most cajacity for the system. The compute servers will be UNIX/POSIX compliant, run Andrew File System (AFS ${ }^{\text {rm }}$ ) and use some type of distributed batch queueing system with network load balancing (e.g., NQS/EXEC).

\subsubsection{File/Print/Security/Administrative Servers}

The File/Print/Security/Administrative servers will also run AFS and will each provide 5-10 Gbyte disk storage. Load balancing, synchronization, redundancy, and concurrence are key administrative functions to be tesied for these servers. The Print Server will route output to the existing network connected devices: e.g., VAX cluster, Laser priniers (Apple), UNIX workstations with directly connected printers. Subsequent upgrades for file storage are planned to include a 10 Gbyte 'Redundant Array of Inexpensive Disks' (R AID).

\subsubsection{Archive/Backup Server}

The Archive/Backup server provides fully automated media handling and serves a dual role by also providing a seemingly infinite onlins storage capacity for backup (500 Gbyte - 1 Tbyte, greatly exceeding the actual online disk storage capacity); a subset of that storage will be used for selective archiving. It is also desirable for this server to support AFS and DCE/DFS. Alternatively, ongoing research at the Pittsburgh 
Supercomputing Center may permit direct use of AFS tc support archiving (see Section 2.5.1).

\subsubsection{Visualization Servers}

A key part of the strategy for scientific computing is to greatly expand the capabilities for scientific visualization as discussed later. Visualization servers are thus a vital part of the SCEED system. These servers will use industry-standard visualization packages such as AVS, Wavefront, and SGI/Explorer. Video hardcopy will be available by using the existing SCS network frame buffer and video recorders.

\subsubsection{User Interface}

Terminal access will be supported by $X$ Window Server and its extensions (PEX). Users will login to SCEED via their existing hardware if it is running an $X$ Window System. Such hardware can be anything from a UNIX workstation to an $X$ terminal to just a personal computer running $X$ terminal emulation software.

\subsection{8 'Wedge' Users}

The SCEED will not be developed separate from the eventual customer and then presented to him as a fait accompli. Instead, a key step for timely success in this project is a commitment of specific personnel who will execute their work on the SCEED. The active involvement of the users will drive the development needs of the system. The SCEED developers will fully commit to implementing the seamless integration of system services to make working in that environment the preferred choice for scientific computation at SRS.

\subsubsection{Status}

The key concept of SCEED is that of a small, robust, production environment that comprises leading edge technology in hardware architecture and software capability. Although, as stated, it will provide a production environment for scientific computing, it is also planned as a development project in which to implement on a test basis a variety of prototypic state-of-the-ant technologies. Such implementations will be done only on selected servers of the system so that the production capabilities of the SCEED are not impaired.

The proposed work is phased. Upgrades discussed above are planned after the initial system has been proved reliable. An Acquisition Plan and Evaluation Benchmarks are being prepared prior to system procurement. In the interim, a Pre-SCEED system is being assembled using existing equipment and software to begin to address system. administration problems, establish protection plans, and use various file servers in such an environment.

\subsection{The Other Parts of the Strategy}

The preceding discussion of the SCEED system is part of a larger strategy, the other components of which are discussed next. If these other components didn't exist, the 
SCEED would be iust a cluster of workstations. While very useful in itself, that would be too limited in scope to provide a long-term comprehensive solution to the computational needs of the site.

The additional necessary components to the long term strategy include: (1) establishing alliances (i.e., cooperative working agreements) in areas of mutual interest pertaining to scientific cumputing with DOE and NSF Supercomputer Centers;

(2) establishing site connection to the Internet, the national high-speed network interconnecting major computing facilities in the country; (3) making available, first through the Internet, and then by acquiring onsite as needed, a massively parallel architecture computer. These items are discussed, in turn, below.

\subsubsection{Alliances with DOE and NSF Supercomputer Centers}

The scope of computational science is too broad, and individual site budgets too limited, for any one site to be able to provide all the required capabilities and developments. 7 Also, the rapidly changing mission of the SRS precludes a major staffing to advance computational science at the site. Further, the Savannah River Technology Center (formerly the Savannah River Laboratory) has always been chartered to be an applications driven technical support and technology transfer organization to the rest of the Savannah River Site. Only where no suitable capability exists elsewhere is a significant research ard development effort undertaken (there have been numerous such cases in the past).

In the computational science arena, it is desired to tap the resources, capabilities, and developments already available at Department of Energy (DOE) and National Science Foundation (NSF) supercomputing centers. DOE has assigned "lead laboratory" or "Center of Excellence" status to specific sites with outstanding expertise in specific areas of computational science. Further, individual universities have obtained both corporate and government support to pursue research in specific areas of high performance computing. Finally, the NSF has established national centers for supercomputer research that provide the latest in both software and hardware for supercomputing applications. At any one time, each center is likely to be the clear leader in a particular area of interest to SRS. All these sources of expertise will be sought to collaboratively and cooperatively obtain the latest developments for implementation in the programs at SRS.

\subsubsection{NSE Supercomputina Centers}

The National Center for Supercomputer Applications (NCSA) is a (if not the') world leader in methods and techniques for the visualization of scientific data and will be the touchstone for SRS applications in this area beyond what is already underway in the SRS Advanced Scientific Computing Environment Team programs. The National Center for Atmospheric Research (NCAR) focuses on applying state-of-the-art

7 Actually, this problem is quite general to the compuing industry. To respond to this problem, companies in all parts of the industry have been increasingly forming alliances with research organizations and/or other companies to develop specific technologies. This trend is also observed in other industries as they strive to be competitive in an environment of increasing competition, increasing costs, and declining resources. 
comciuting facilicies to atinospheric and ocean sciences research. (Atmospheric transport of radionuclides is of intense interest to SRS. A major effort has been underway here for years to improve the site's capability to predict the transport path of, and define appropriate emergency response to, postulated accidental releases.) The Center for Supercomputing Research and Development at the University of Illinois, Urbana-Champaign, (CSR ! is a leader in research on compilers for automatic parallelization of application codes and in developing methodologies and measurement tools to understand the process of speeding up application programs. A joint effort to parallelize SRS thermal hydraulics, environmental transport, and physics applications codes is being discussed, as well as applying CSRD expertise to review SRS proposed program directions and provide oversight of our overall strategic plan for high performance computing.

The Pittsburgh Supercomputing Center (PSC) is a leader in use of, and extensions to, the AFS $^{\text {im }}$ which is the distributed file system initially to be used with the SCEED s:; stem (see Section 2.4). The PSC has reported on extensions to AFS that permit: (1) use in Cray supercornputer environments running UNICOS (Cray's Unix operating system), and (2) multiple residency to achieve transparent archival capability. ${ }^{8} W_{\theta}$ plan to investigate incorporating these AFS extensions into the SCEED system.

Mechanisms are already established by the individual NSF Supercomputer Centers to disseminate, and facilitate use of, their respective developments in computational science. For example, the NCSA has three types of partnerships designed to meet individual needs of an oreanization. Of these, the Advanced Partnership is available for organizations such as SRS that have extensive corrputational science experience. This type of partnership is very flexible and offers opportunities for specifically targeted support in various components of high-performance computing. Such a partnership is highly desirable and is an important part of our plan to satisfy SRS's continually changing computational needs.

\subsubsection{DOE Supercomoutine Centers}

Los Alamos National Laboratory (LANL) has been since its beginning, and continues to be, a leader in computational science. LANL, which provides an open environment of a variety of advanced ano novel computer architectures probably urmatched elsewhere, has been facilitating supercomputing at SRS for several years. (Prior to SRS obtaining its own Cray XMP-EA, access to the LANL array of supercomputers was available through a datalink to that site.) It is expected that this interaction will expand to a more collaborative effort. The Advanced Computing Laboratory of LANL is also affiliated with the NSF Center for Research on Parallel Computation at Rice University. The latter is pursuing developments in software architecture to facilitate methods in parallel computing.

Lawrence Livermore National Laboratory (LLNL), also a pioneer in supercomputing, has recently initiated a project to use a client-server network of High Performance Workstations to serve as a major computational resource and with which to pursue

8 D. Nydick, of al. "An AFS ${ }^{m}$ Based Mass Storage System at the Pittsburgh Supercomputing Center." Eleventh IEEE Symposium on Mass Storage Systems, October 7-10, 1991, pp. 117-122, 
studies of distributed and parallel computing. (The SRS SCEED system described above is a variant of the LLNL project.) Sandia National Laboratory (SNL), Oak Ridge National Laboratory (ORNL), and Argonne National Laboratory (ANL) also have significant programs designed to advance various aspects of high performance computing.

Collaborative efforts with the DOE National Laboratories have been continual at SRS since the latter's founding. These efforts have ranged from early critical experiments on potential reactor lattice configurations to reviews of safety analyses and risk assessments to providing supercomputer cycles as discussed above. Such interaction will continue within the DOE community and focus particularly on the targeted areas of high-performance computing (distributed computing, parallel computing, meta-computer development, etc.) and scientific viswalization.

\subsubsection{Site Connection to the Internet}

The Research Internet is a national system of interconnected networks linking most government sites including the DOE National Laboratories and NSF Supercomputer Centers discussed above, universities, and many cor ir ations including IBM and DEC. The Internet is used by its participants as a means of electronic messaging, publication, and sharing of data, and to obtain access to computing facilities beyond these available locally.

Establishing an Internet connection and buildiny a network authentication service are seen as absolutely necessary to establish SRS as a model of excellence in the DOE community. Connection of the SRS Network will facilitate interchange with the broad technical community and enhance opportunities for the collaborative research and development envisioned here. Also, SRTC is responsible for establishing coordinated research with the South Carolina and Georgia university consortia (SCUREF and ERDA, respectively). The primary tie tu this consortium will be via the Southeastern Universities Research Association net (SURAnet). This latter network is connected to the Research Internet.

Beyond the vast information available through the Internet, access to supercomputing cycles is also available from various sites via the Internet connection. As discussed below (Section 2.5.3), the technology of parallel computation promises to allow increasingly sophisticated analyses to be completed fast and cheaply enough to consider for a greater proportion of the SRS seismic, environmental, thermal hydraulic, Monte Carlo physics, etc., studies. (The demand for the sophisticated analyses has almiays existed: the required technology has not .) Thus, an important part of any stratigy for scientific computing must include studies in parallel computation because it has the potential to completely revamp scientific computing strategies.

Proliminary studies of the applicability of parallel computing on distributed platforms for SRS codes can be done onsite via the SCEED project discussed above. Also, limited studies of parallel computing using up to four local processors can be done with the IBM 3090 onsite. However, studies of the usefulness and applicability of, and productivity gains to be obtained with, massively parallel architecture can not now be done onsite. We plan to do supporting studies on a variety of massively parallel. 
machine architectures (SIMD, MIMD, and shared memory system: see Section 2.5.3). These machines will initially be accessed at various supercomputer centers (see Section 2.5.1) via the internet connection. This will permit sufficient analyses to be done to support ongoing hardware acquisition plans to provide necessary upgrades in computational capability for SRS.

These studies will also guide the continual development of software to support site scientific computational needs. The use of the Internet connection will permit these initial studies to be completed without expenditure of capital funds. The studies of scientific computation via the Internet will also indicate the feasibility of obtaining all needed sprint capacity for computing cycles from that mode. The trend in technology of supercomputing, forecast long ago, is clearly that "computing utility companies" will be a prime source of high-performance cycles, just as electric utilities are the source of electricity to almost every home in the country. Such computational utilities can be an efficient, low-cost supplier of computing cycles. It is possible to consider keeping only minimal capacity (relatively) onsite to meet routine needs. This approach meets the need for flexibility to respond to changing mission, budget, and technology discussed previously (Section 2.2).

The strategy defined here is entirely consistent with the plan currently being drafted by the Defense Department to consolidate and modernize its supercomputing sites. ${ }^{9}$ That plan recommends consolidating DOD's supercomputing resources at fewer regional supercomputer centers that will support all service branches via high-speed networks to some of the smaller, more remote laboratories. That plan also recommends increased sharing of DOD supercomputer resources.

\subsubsection{Massively Parallel Architecture Computer}

The computer vendors and the computer research communities agree that the next generation supercomputers will be based on parallel architectures. Figure 2 shows one view of a hierarchy of historical and present computational parallel architectures.

SRS is preparing for the transition from conventional to parallel architectures with the following phased steps:

(1) collaborations with knowledgeable researchers in the field (se日 Section 2.5.1);

(2) employing networks of workstations with research parallel software on coarse-grained, loosely coupled parallel problems (the SCEED system would be the environment for this application; see Section 2.4);

(3) obtain offsite access to massively parallel machines (Thinking Machines, NCube, Intel, Kendall Square Research) for investigation of coarse- and fine-grained, tightly coupled parallel problems (via the Internet; seo Section 2.5.2);

4) obtain a small parallel processing research machine at SRS for direct work with the full range of internal codes and data; and,

9 C. D. Marsan. "DOD Plans to Merge and Purge Super Sites.", Federal Computer Week, Vol. 6, No. 8, p.1 (April 13, 1992) 
(5) SRS supercomputer capability circa FY-96 which will almost certainly be a parailei machine (either hybrid scalar/parallel or fully parallel).

The platforms for items 4 and 5 would most probably be of scalable design in keeping with the scalability of the SCEED system discussed previously (see Section 2.4).

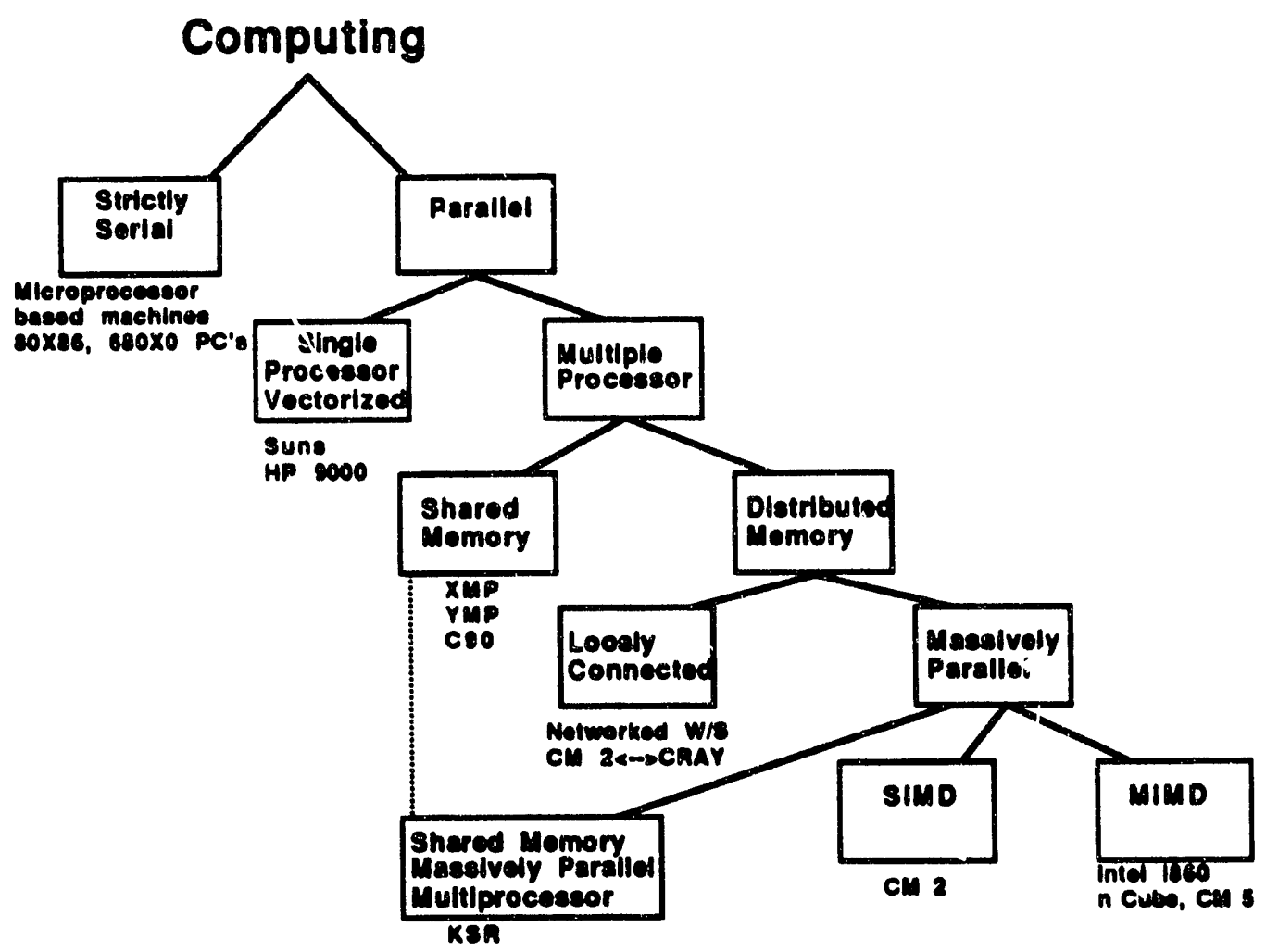

Figure 2. Computing Architectures

\subsubsection{Programmatic Need}

The areas of computing that have historically placed the greatest demand on SRS computing resources include reactor physics, reactor thermal-hydraulic analyses, reactor safety analyses, and accident management. Recent demands for power limits calculations, PRA model upgrades, and severe accident analyses have taxed available hardware and software capabilities. Increased complexity of charges for special isotope production would place further demands on the systems. Although some of these reactor programs are decreasing as the site mission changes, the computational needs associated with nuclear waste management, and environmental protection and remediation are requiring more complex engineering and physics analyses. A crucial step in all these activities is improved visualization capabilities to facilitate rapid understanding and interpretation of computational and experimental results. 
The following paragraphs describe the requirements of some of the above mentioned activities. To meet these requirements SRS must move to the next generation of supercomputers with vastly increased total performance and capacity. While not optimum for meeting all the requirements of the above mentioned activities, parallel computing will be a significant component in many of them. Clearly, SRS must have access to the resources necessary to understand how to apply parallel architectures to those areas classically treated with scalar or vectorizing techniques. This understanding is strategic to SRS programs.

\subsection{Physics Calculations}

The entire area of reactor physics, nuclear criticality, and radiation shielding calculations lends itself particularly well to improving the modeling via use of parallel computing methods. The most obvious example is in the area of Monte Carlo calculations. Such calculations compute the probabilistic history of neutrons and/or gamma radiation, for example, from birth to death throughout complex geometric and nuclear reaction environments. Each such history is statistically independent and can be computed in parallel with all other histories. The full capability of a massively parallel computer could be used to provide greatly increased speed for this type of calculation. And because Monte Carlo calculations have the potential to be much more accurate than standard techniques, the ability to routinely use them for more types of physics and engineering calculations offers the promise of a major increase in our assessment of conditions affecting reactor and nonreactor nuclear safety as well as improving the accuracy of predicting actual production of isotopes and thus the actual content of process waste.

\subsection{Environmental Program Calculations}

SRS does axt6nstive modeling of surface water (stream, swamp, river) and groundwater flow and contaminant transport to support a variety of activities. The simulation results from the modeling are used to determine the fate of environmentally released constituents, to estimate the effects of groundwater usage on the subsurface water resource, to evaluate engineered waste management facilities (e.g. saltstone facility), and to evaluate environmental impacts to meet regulatory requirements. Increasingly more sophisticated models are required to evaluate the effectiveness of proposed remediation programs and to determine whether such programs can satisfy the regulatory requirements and desired goals. Additional areas of computational interest that overlap physics calculations, above, include accident management studies to predict atmospheric transport of postulated accidental releases of gaseous radionuclides. Such calculations would be used to aid studies to support and develop procedures for emergency evacuations from the site and surrounding environs.

It is expected that this growth in environmental studies will continue at an ever faster pace and that the demands for visualization of the results of $3 D$ modeling of groundwater and underground aquifer flows will require vastly increased supercomputing resources. 


\subsubsection{Supercomouter Uparade}

The selection of a particular implementation of supercomputer architecture for parallel computing (i.e. single Instruction-multiple data (SIMD), multiple instruction-multiple data (MIMD), et al.) with which to upgrade the sites mainframe facilities can not, and need not, be defined at this time. ${ }^{10}$ Most probably, however, it will be scalable so that subsequent upgrades could be done in reasonable increments driven by application demand.

The selection of architecture will be made after a thorough analysis of need coupled with extensive use of all candidate architectures via the Internet access to offsite supercomputer centers. A key factor in choosing the upgrade architecture will be its applicability to the environment then being developed with the SCEED system. Open, transparent seamless integration of facilities will be a major factor in the final selection. While there may be several, technically valid, architectures or vendor models that will satisfy the application requirements, there are unnatural constraints imposed by the procurement process that may eliminate many of these. The final selection will be a compromise of the best technical solution that also meets the DOE procurement procedures.

10 It is inappropriate to speak of upgrading SRS 'supercomputing' facilities because we have no supercomputer. The vector processing mainframe, namely the Cray XMP.EA, has only one processor. While fast, the machine can not be considered to be a supercomputer by today's standards. Rather, it is simply a vectol' processing mainframe providing very necessary cycles and is presently running at full capacity. 

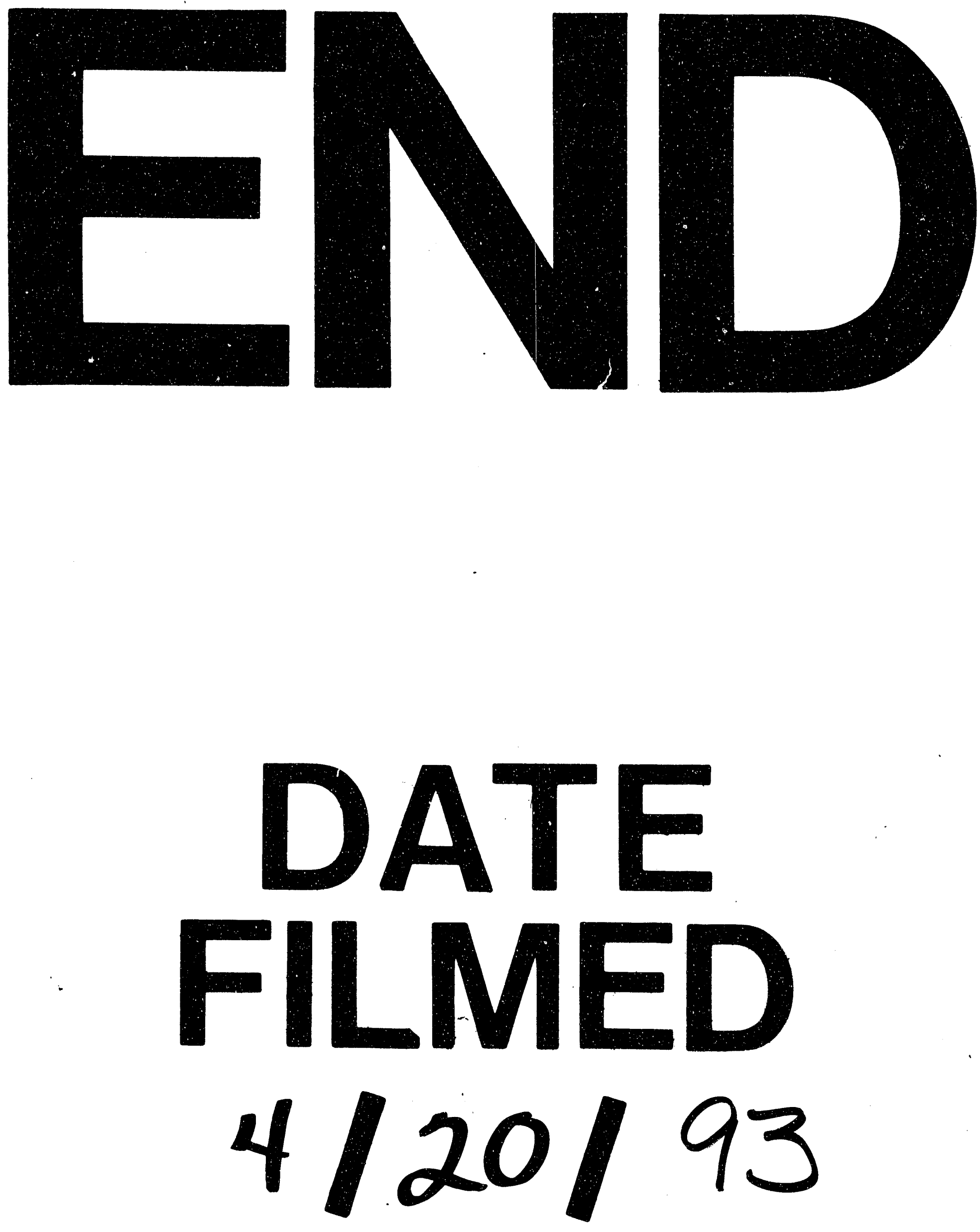

1 
